\title{
Two-pore channel 2 is a key regulator of adipocyte differentiation via the cAMP signaling pathway with calpain as downstream effector
}

Authors: Yuxuan Zhang ${ }^{1} \dagger$, Lai-Hing Chan ${ }^{1} \dagger$, Ruth Tunn ${ }^{1} \dagger$, Margarida Ruas ${ }^{1}$, David Gay $^{1}$, Marijana Todorcevic ${ }^{2}$, Costas Christodoulides ${ }^{2}$, John Parrington ${ }^{1 *}$

Affiliation: ${ }^{1}$ Department of Pharmacology, University of Oxford, United Kingdom, OX1 3QT; ${ }^{2}$ Oxford Centre for Diabetes, Endocrinology and Metabolism, University of Oxford, United Kingdom, OX3 7LE.

*Corresponding author: John Parrington john.parrington@pharm.ox.ac.uk

$\dagger$ These authors contributed equally

\section{Key words}

Adipocyte differentiation / Calpain / CaMKII / cAMP / CREB phosphorylation / Two pore calcium channel (TPC). 


\begin{abstract}
We investigated whether the endolysosomal two-pore channel TPC2 is a mediator of adipocyte differentiation. We show that Tpcn 2 mRNA is expressed transiently during induction of $\mathrm{C} 3 \mathrm{H} 10 \mathrm{~T} 1 / 2$ mesenchymal stem cells to differentiate into adipocytes, and that this expression is triggered by cAMP. This is the first demonstration of a cell signaling pathway that can regulate TPC gene expression. We also identified an important functional role for TPC2 in adipocyte differentiation. First, ectopic TPC2 expression in $\mathrm{C} 3 \mathrm{H} 10 \mathrm{~T} 1 / 2$ cells partially rescued the block to adipocyte differentiation caused by cAMP absence. Second, inhibition of endogenous TPC 2 expression in primary preadipocytes substantially reduced their ability to differentiate into adipocytes. Finally, genetic variation at the Tpcn2 locus is associated with increased upper-body fat distribution in women concomitant with reduced Tpcn 2 expression in abdominal adipose tissue. Our findings implicate TPC2 as an important mediator of adipogenesis and may aid identification of new drug targets for treatment of obesity.
\end{abstract}

\title{
Introduction
}

The rise of obesity in the developed and developing world and the increased risk of type 2 diabetes, coronary heart disease, stroke, and cancer that accompanies this condition, is leading to increased interest in adipogenesis, the process by which adipose progenitors differentiate into mature adipocytes (Sarjeant \& Stephens, 2012). Adipose tissue is a dynamic organ composed of adipocytes which are the predominant cell type, and a variety of other cell types such as preadipocytes, endothelial cells, macrophages and some immune cells (Sarjeant \& Stephens, 2012). Expansion of adipose tissue is led by increases in both the number and size of adipocytes (Ali et al, 2013). On the one hand, increasing synthesis and storage of triacylglycerols (TAGs) in adipocytes increases the size of these cells, which is defined as hypertrophic adipose tissue growth; on the other hand, differentiation from fibroblast like preadipocytes increases the number of adipocytes, which is referred to as hyperplastic adipose tissue growth and also adipogenesis (Engin, 2017). Adipogenesis includes two phases: first, pluripotent mesenchymal stem cells develop into preadipocytes and thereby become committed to the adipogenic lineage; second, these preadipocytes differentiate into lipid-laden adipocytes. The signaling mechanisms underlying preadipocyte determination and the early stages of adipocyte differentiation remain poorly characterized; thus deciphering these mechanisms could have important implications for the treatment of obesity and related conditions by identifying signaling proteins that could be targeted in people with these disorders. Changes in intracellular calcium $\left(\mathrm{Ca}^{2+}\right)$ constitute a universal signaling mechanism that mediates a variety of important physiological processes (Berridge et al, 2003). However, the role of $\mathrm{Ca}^{2+}$ signals as mediators of adipocyte differentiation still remains far from clear. Thus pharmacologically induced $\mathrm{Ca}^{2+}$ changes have been shown to both stimulate and inhibit adipocyte differentiation in vitro, depending on the point in the differentiation process at which such changes were induced (Ntambi \& Takova, 1996); however, one potential problem with such approaches is that they are unlikely to mimic the complex spatiotemporal $\mathrm{Ca}^{2+}$ signaling patterns that may be occurring during endogenous differentiation. In terms of downstream effectors of $\mathrm{Ca}^{2+}$ signals during adipocyte differentiation, the $\mathrm{Ca}^{2+}$ - activated protease calpain has been implicated in adipogenic differentiation of preadipocyte cells. Thus calpain mRNA 
levels were shown to be increased during the first few days of adipogenesis, and inhibition of this protease repressed differentiation by preventing c/EBP $\beta$ binding to the promoter of the $\mathrm{c} / \mathrm{EBP} \alpha$ gene (Patel \& Lane, 1999). Other studies have suggested that the $\mathrm{Ca}^{2+}$ activated kinase CaMKII (Zhang et al, 2018), and the $\mathrm{Ca}^{2+}$ activated phosphatase, calcineurin (Liu \& Clipstone, 2007; Neal \& Clipstone, 2002), may both play roles in adipocyte differentiation. However, the source of the upstream $\mathrm{Ca}^{2+}$ signal(s) that is regulating these downstream effectors during adipocyte differentiation remains to be identified. One important way in which $\mathrm{Ca}^{2+}$ signals are generated is through release of $\mathrm{Ca}^{2+}$ from intracellular stores by the action of $\mathrm{Ca}^{2+}$ mobilizing second messengers. The three primary $\mathrm{Ca}^{2+}$ mobilizing messengers are inositol trisphosphate $\left(\mathrm{IP}_{3}\right)$, cyclic ADP ribose (cADPR), and nicotinic acid adenine dinucleotide phosphate (NAADP). It has been known for some time that $\mathrm{IP}_{3}$ and cADPR release $\mathrm{Ca}^{2+}$ from the endoplasmic reticulum (ER) via $\mathrm{IP}_{3}$ receptors $\left(\mathrm{IP}_{3} \mathrm{Rs}\right)$ and ryanodine receptors (RyRs), respectively (Galione \& Chuang, 2020; Galione \& Churchill, 2000). In contrast, NAADP targets a $\mathrm{Ca}^{2+}$ store located in endolysosomelike acidic organelles (Galione, 2011). Importantly, there appears to be a complex interrelationship between these three signaling pathways, with NAADP acting to induce local $\mathrm{Ca}^{2+}$ release from acidic organelles that can then act as a 'trigger' to stimulate global $\mathrm{Ca}^{2+}$ release from the ER via $\mathrm{IP}_{3} \mathrm{Rs}$ and RyRs (Galione \& Churchill, 2002), but also recent evidence indicating that this relationship is bidirectional. Thus, we have recently shown that $\mathrm{Ca}^{2+}$ released from the ER can activate the NAADP pathway both by stimulating $\mathrm{Ca}^{2+}$-dependent NAADP synthesis and by activating NAADP-regulated $\mathrm{Ca}^{2+}$ channels (Morgan et al, 2013). The identity of such NAADPregulated $\mathrm{Ca}^{2+}$ channels remains controversial. In line with their assumed location in acidic organelles, studies by ourselves and others have identified the two pore channels (TPCs; gene names Tpcn1 and Tpcn2) as endolysosomal ion channels and integral components of the intracellular receptor for NAADP (Brailoiu et al, 2009; Calcraft et al, 2009a; Ruas et al, 2010; Jin et al, 2020; Ruas et al, 2015). Moreover, gene knockout (KO) of TPC1 and TPC2 in mouse embryonic fibroblasts abolished their ability to mediate NAADP-regulated, $\mathrm{Ca}^{2+}$ signaling responses (Ruas et al, 2015). In this study, we investigated the role of TPC2 during adipogenesis, both by studying adiposity in TPC2 $\mathrm{KO}$ mice, and by investigating the pattern of expression and functional role of TPC 2 during adipocyte differentiation in vitro and by investigating the association between genetic variants at TPCN2 and body fat distribution in humans.

\section{Results}

\section{TPC2 KO mice of both sexes have abnormal adiposity compared to wild type controls}

To determine whether there were any differences in fat or lean content of Tpcn $2 \mathrm{KO}$ mice, mice of both sexes of this strain were analysed using time domain nuclear magnetic resonance (TD-NMR), at 3, 6 or 9 months of age. Male Tpcn 2 KO mice were significantly less heavy than wild type (WT) controls at all ages studied, mainly due to lower fat mass. Lean mass was not significantly different in Tpcn $2 \mathrm{KO}$ males, except in the 9 month old cohort, in which significantly reduced lean mass was observed for Tpcn $2 \mathrm{KO}$ males. This translated into an overall reduced body fat percentage with concomitant increase in percentage of lean composition (Fig. S1A). Tpcn $2 \mathrm{KO}$ females were also significantly lighter than WT controls, up to 6 months, 
and similar to the male group this was due to reduced fat mass. The reduced fat mass and normal lean mass seen in 3- and 6-month-old females resulted in a decreased body fat percentage and increased percentage in lean composition. In contrast, the 9month Tpcn $2 \mathrm{KO}$ female group showed no significant differences in any of the parameters measured, when compared to the WT controls (Fig. S1B). To further investigate the physiological condition of Tpcn2 $\mathrm{KO}$ mice, an intraperitoneal glucose tolerance test (IPGTT) was carried out (Fig. S2). No differences in the response to a glucose challenge were detected between Tpcn2 KO mice and WT controls.

\section{TPC2 mRNA expression is up-regulated transiently during adipocyte differentiation in vitro}

Differences in adiposity in TPC2 KO mice in vivo might have a number of different underlying causes. In this study we decided to focus on whether TPC2 might be involved in one specific process linked to adiposity, namely adipocyte differentiation. To see whether TPC2 might play a role in adipocyte differentiation in vitro, we used the $\mathrm{C} 3 \mathrm{H} 10 \mathrm{~T} 1 / 2$ cell line, a mesenchymal stem cell line that can differentiate along adipogenic, myogenic and chondrogenic pathways (Taylor \& Jones, 1979). In the presence of a cocktail of insulin/insulin-like growth factor 1, dexamethasone and IBMX in serum-containing medium, C3H10T1/2 cells differentiate into adipocytes. Progression of adipogenesis was monitored by Oil Red O staining or AdipoRed fluorescence (Fig. 1A), which both measure accumulation of lipid droplets, and RTqPCR analysis of mRNA expression of adipogenic markers (Fig. 1B), to ensure that the morphological changes observed in cells subjected to the standard differentiation protocol were indicative of true adipogenesis. Early transient upregulation of $c / E b p \delta$ mRNA expression, followed by sustained Ppary 2 and Srebp 1 mRNA expression and later $a P 2$ mRNA expression, which is the expected sequential pattern of expression of these genes during adipocyte differentiation, confirmed adipogenesis at the molecular as well as the morphological level (Fig. 1B). We next studied whether Tpcn 2 mRNA expression levels changed during the differentiation process; notably, these increased significantly early during adipocyte differentiation, and in a transient manner, with Tpcn 2 mRNA levels peaking at around 4 hours post-induction and returning to starting levels by around 48 hours after the start of induction (Fig. 1C).

\section{Tpcn $2 \mathrm{mRNA}$ expression during adipocyte differentiation is up-regulated by a cAMP signal}

To investigate which components of the adipocyte differentiation cocktail were responsible for the observed changes in Tpcn $2 \mathrm{mRNA}$ expression, C3H10T1/2 cells were treated with the individual components separately, and harvested at 4 hours postinduction, a stage at which significant change in expression was previously seen. Tpcn 2 mRNA levels were significantly increased by IBMX, to a level comparable to that achieved by complete differentiation medium, whilst the other components of the cocktail had no significant effect on Tpcn2 expression (Fig. 2A). Moreover, treatment with medium lacking IBMX but containing all other components of the differentiation cocktail, failed to induce Tpcn $2 \mathrm{mRNA}$ expression (Fig. 2B). This finding suggested a potential involvement of cAMP signaling in the induction of Tpcn2 expression, since IBMX inhibits cAMP phosphodiesterase (PDE), and thus acts to increase cAMP levels (Thompson, 1991). To further confirm the involvement of the cAMP signaling pathway in the induction of Tpcn 2 gene expression, alternative methods of raising levels of cAMP were used to investigate whether this is the signaling intermediate by which IMBX affects Tpcn2. Rolipram, like IBMX, is a cAMP PDE inhibitor; 
however, while IBMX is relatively nonspecific and may affect cGMP as well as cAMP levels, rolipram is a specific inhibitor of PDE IV, thus it specifically raises cAMP levels (Thompson, 1991). In contrast, forskolin increases cAMP levels by activating the synthesising enzyme adenylyl cyclase (Laurenza et al, 1989). We found that both rolipram and forskolin increased Tpcn 2 mRNA levels after 4 hours treatment (Fig. 2C). The increase for both drugs was similar to, though slightly lower than, that seen with IBMX. Further evidence for a role of cAMP in mediating IBMXinduced Tpcn 2 mRNA upregulation was provided by our finding that pretreatment with the adenylyl cyclase inhibitor SQ22536 significantly reduced this IBMX-induced rise in expression (Fig. 2D) (Haslam et al, 1978).

Our finding that the cAMP signaling pathway is involved in the regulation of Tpcn2 gene expression during adipocyte differentiation raises the question of which proteins that are downstream of cAMP in this pathway mediate these effects. Previous studies have shown that cAMP can activate protein kinase A (PKA), or alternatively exchange protein activated by cAMP (Epac) (Cheng et al, 2008). To investigate the possible involvement of PKA and Epac in the induction of Tpcn2 gene expression, we studied whether inhibitors of these proteins had an impact on such expression. We found that PKI, an inhibitor of PKA, significantly inhibited Tpcn 2 mRNA expression 2 hours after induction of adipocyte differentiation (Fig. 2E). Treatment with ESI-09, an Epac inhibitor (Ahmed et al, 2019), significantly inhibited Tpcn 2 mRNA expression at 2 hours and this effect continued to the 4 hour time point (Fig. 2F). PKA and Epac can both mediate gene expression via the transcription factor cAMPresponse element binding protein (CREB) (Kelly, 2018). To study whether CREB plays a role in the CAMP-induced expression of the Tpcn2 gene, we investigated the effects of a CREB inhibitor, 666-15 (Xie et al, 2019). We found that this significantly reduced the expression of Tpcn 2 mRNA during adipocyte differentiation (Fig. 2G). Our findings suggest that both PKA and Epac may be involved in the cAMP-mediated regulation of Tpcn2 gene expression, via a mechanism involving CREB.

\section{TPC2 plays a functional role during adipocyte differentiation in vitro}

The striking transient upregulation of Tpcn 2 mRNA expression during differentiation of $\mathrm{C} 3 \mathrm{H} 10 \mathrm{~T} 1 / 2$ cells into adipocytes in vitro, suggests that TPC2 may be playing a functional role in this process. We investigated this possibility initially by ectopically expressing recombinant TPC2 protein in $\mathrm{C} 3 \mathrm{H} 10 \mathrm{~T} 1 / 2$ cells; thus, we used a lentiviral vector to express mCherry-tagged TPC2, with mCherry alone used as a control. Expression of mCherry or mCherry-tagged TPC 2 in infected cells was confirmed by immunoblotting with an anti-mCherry antibody (Fig. 3A), and by analysis of mCherry fluorescence and anti-mCherry immunofluorescence. (Fig. 3B; Fig. S3)

The effects of heterologous TPC2 expression on adipogenic differentiation was assessed by measuring AdipoRed fluorescence (Fig. 3C). Lentiviral-mediated expression of TPC 2 did not affect adipogenic differentiation of $\mathrm{C} 3 \mathrm{H} 10 \mathrm{~T} 1 / 2$ cells in response to the standard differentiation protocol. However, when IBMX was omitted from the differentiation medium, this led to almost complete suppression of adipogenesis, but if TPC2 was expressed virally, cells exhibited significantly higher levels of differentiation than mCherry-infected controls (Fig. 3C). In addition, preadipocyte cells were isolated from WT or Tpcn $2 \mathrm{KO}$ mice and induced to differentiate into adipocytes. While cells isolated from WT mice showed a high degree of differentiation, those from Tpcn2 KO mice were inhibited in their differentiation capacity to a significant degree (Fig. 3D).

\section{TPC2 regulation of adipocyte differentiation is mediated by calpain}


Our finding that TPC2 appears to be playing a functional role in adipocyte differentiation in vitro raises the question of the mechanism by which it does this. Given the evidence implicating TPC2 as an NAADP-regulated $\mathrm{Ca}^{2+}$ channel, one possibility is that a $\mathrm{Ca}^{2+}$-regulated effector protein downstream of TPC2 is involved in the differentiation of preadipocytes into adipocytes. Calpain is a $\mathrm{Ca}^{2+}$-dependent cytosolic protease that binds to membranes on its activation and proteolyses substrate proteins in a specific manner; a previous study showed that calpain is required for differentiation of 3T3-L1 preadipocytes into adipocytes (Patel \& Lane, 1999). Interestingly, this same study showed that calpain expression is induced by changes in cAMP levels, just as we have shown in this current study for TPC2. To investigate whether calpain activity is regulated by $\mathrm{Ca}^{2+}$ signals mediated by TPC2, we studied changes in calpain activity during differentiation of primary preadipocytes obtained from WT or Tpcn $2 \mathrm{KO}$ mice, into adipocytes. For the WT preadipocytes, what appears to be a gradual rise in calpain activity after induction of differentiation up to 8 hours, is followed by an approximately three-fold spike in activity at 24 hours, after which activity decreases and levels off by 4 days (Fig. 4A). In contrast, for the Tpcn2 KO preadipocytes, we saw a gradual increase in calpain activity that continues up to 8 days, but no sign of a transient burst of activity at around 24 hours as was seen for the WT controls (Fig. 4B). This indicates that calpain activity during adipocyte differentiation is substantially reduced by $T p c n 2 \mathrm{KO}$, in a qualitatively distinct manner, and suggests that calpain is an effector of TPC 2 in this process.

\section{CREB phosphorylation during adipocyte differentiation is regulated by TPC2}

Our finding that Tpcn 2 mRNA expression is regulated by cAMP/PKA/Epac/CREB implies that TPC2 is downstream of CREB during adipocyte differentiation. However, there are often reciprocal interactions between components in signaling pathways. We therefore decided to investigate whether Tpcn2 $\mathrm{KO}$ had any effect on the active, phosphorylated state of CREB during adipocyte differentiation, using an ELISA assay to compare levels of total, and phosphorylated, CREB, during differentiation of primary preadipocytes from WT or Tpcn $2 \mathrm{KO}$ mice, into adipocytes. This analysis showed that compared to a steadily increasing phosphorylation level of CREB in WT preadipocytes, CREB phosphorylation was inhibited in Tpcn $2 \mathrm{KO}$ preadipocytes after approximately 1-2 days following induction of differentiation, although the Tpcn $2 \mathrm{KO}$ preadipocytes had an initially higher level of phosphorylated CREB than WT preadipocytes up to day 1 of the differentiation process (Fig. 5B). $\mathrm{Ca}^{2+} /$ calmodulin-dependent protein kinase (CaMK) triggers phosphorylation of CREB by complex mechanisms (Aimes et al, 2000; Ma et al, 2014). To investigate whether TPC2 affects the phosphorylation of CREB via a mechanism involving CaMK, we studied the effects of KN 93, a selective CaMKII inhibitor (Bonnefond et al, 2015). We found that treatment with this inhibitor leads to an inhibition of CREB phosphorylation, particularly after day 2 of differentiation, in a way that partially mimicks the effects of Tpcn $2 \mathrm{KO}$ (Fig. 5C,D). This suggests that TPC2 may normally be playing a role in enhancing CREB phosphorylation during adipocyte differentiation, via a mechanism involving CaMKII (Fig. 7).

Genetic variation at the TPCN2 locus is associated with altered fat distribution. To determine the relevance of our findings to humans we examined the tissue expression pattern of TPCN2 in GTEx (GTEx Portal). We found that among metabolic organs and tissues, abundance of TPCN2 mRNA was highest in adipose tissue (Fig. 6A). Furthermore, based on a survey of RNA sequencing data from 
fractionated adipose tissue from 11 females, we found that TPCN2 expression was highest in stromovascular cells compared with mature adipocytes (Fig. 6B). We next interrogated data from the GIANT consortium and UK Biobank combined genomewide association study (GWAS) meta-analysis (Pulit et al, 2019). This analysis revealed that an independent signal at the TPCN2 locus was associated with waist-hip ratio adjusted for body mass index (WHRadjBMI) specifically in females (beta $=$ 0.0208, $\mathrm{p}=6.06 \mathrm{E}-11$ ) (Fig. 6C). No association was detected in males (beta $=0.0019$, $\mathrm{p}=0.6$ ). The lead single nucleotide variation (SNV) at this signal ( $\mathrm{rs} 2305498)$ lies within an intron of TPCN2 and is in high mutual linkage disequilibrium (LD) with a rs66958567 $\left(\mathrm{r}^{2}=0.79\right)$ which is a strong expression quantitative trait locus (eQTL) for TPCN2 in visceral (NES $=0.34, \mathrm{p}=5.6 \mathrm{E}-13)$ and subcutaneous thigh adipose tissue (NES $=0.32, p=8.8 \mathrm{E}-11$ ) based on data from GTEx (https://gtexportal.org). The same LD block also contains a missense variant in TPCN2 (rs35264875) although this is not predicted to be deleterious by PolyPhen and SIFT. Notably, the WHRadjBMI increasing allele at this signal is associated with lower adipose TPCN2 expression consistent with the pro-adipogenic effect of mouse Tpcn 2 in vitro. Collectively these data suggest that TPCN2 plays an important and sexually dimorphic role in adipose tissue biology and fat distribution in humans.

\section{Discussion}

In this study we explored for the first time the link between the two-pore channel, TPC2, and adipogenesis. Our interest was initially stimulated by the observation that Tpcn $2 \mathrm{KO}$ mice of both sexes have both a reduced absolute fat mass and a reduced percentage of fat at 3 and 6 months compared to wild-type controls. These findings show that elimination of Tpcn 2 expression has an effect on adiposity in mice. These findings raise the question of how loss of Tpcn 2 expression might give rise to such an effect. One problem in pursuing this issue further mechanistically is that with such a whole animal KO, loss of Tpcn 2 expression may have various effects due to changes in the properties of a number of different cell types/tissues, some of which may oppose each other. In addition, a common phenomenon in mice KOs is the occurrence of compensation for loss of expression of the knocked-out gene by changes in the levels and patterns of expression of other genes. This complicates interpretation of what a KO phenotype may reveal about the normal physiological role of a gene in vivo. We thus chose in the remainder of this study to focus on one potential function for TPC 2 in the regulation of the related phenomena of adiposity and adipogenesis, by investigating whether TPC2 plays a role in adipocyte differentiation in vitro. This study provides the first demonstration of a specific cell signaling pathway that can affect the expression of a member of the TPC gene family (Tpcn 2 in mice; TPCN2 in humans). We found that changes in cAMP levels lead to changes in Tpcn2 mRNA expression and previous studies have shown that cAMP can activate protein kinase A (PKA), or alternatively exchange protein activated by cAMP (Epac), both of which can work via CREB (Cheng et al, 2008). Our findings using specific inhibitors of these proteins suggest that both PKA and Epac may be involved in the cAMPmediated regulation of Tpcn 2 gene expression, and demonstrate a direct role for CREB in this process (Fig. 7).

That TPC2 may play a functional role during adipocyte differentiation, is suggested by two main findings in the current study. First, we show that over-expression of TPC2 in C3H10T1/2 cells can partially overcome the block to adipocyte differentiation due to the absence of IBMX from the differentiation cocktail. It 
remains to be shown why this 'rescue' is only partially effective; one possible explanation could be that IBMX induces other changes that are necessary for differentiation to occur so that TPC2 expression on its own is only partially able to substitute for absence of cAMP signaling. Second, we show that loss of endogenous TPC2 function in primary preadipocytes from Tpcn2 KO mice substantially inhibits the ability of these cells to differentiate into adipocytes.

Since previous studies by ourselves and others have previously identified TPCs as integral components of the NAADP-regulated $\mathrm{Ca}^{2+}$ signaling pathway, we chose to study whether the downstream mediator of TPC2 action during adipocyte differentiation might be calpain, since this $\mathrm{Ca}^{2+}$-sensitive protease has previously been shown to be required for differentiation of 3T3-L1 preadipocytes into adipocytes (Patel \& Lane, 1999). The fact that Tpcn2 KO only appears to affect part of the change in calpain activity during adipocyte differentiation, namely that which occurs transiently at around 24 hours after the start of the differentiation process, is interesting given that Tpcn 2 mRNA expression also occurs transiently, but in this case peaks at 4-8 hours after the induction of differentiation. One possible explanation for these findings would be that there are multiple calpain isoforms that are active during the differentiation of preadipocytes to adipocytes, but only one is regulated by $\mathrm{Ca}^{2+}$ signals that are generated via TPC 2 . A previous study suggested that $\mu$-calpain rather than m-calpain was responsible for the control of adipogenesis of ST-13 preadipocytes established from adult primitive mesenchymal cells, through the regulation of the levels of PPARs and C/EBP (Yajima et al, 2006). It would be interesting therefore in future studies to investigate whether a specific calpain isoform is being activated by a $\mathrm{Ca}^{2+}$ signal originating from TPC 2 and whether this isoform is $\mu$-calpain, since this has been specifically implicated in adipocyte differentiation. In addition, we also showed that the CREB phosphorylation level was enhanced by Tpcn $2 \mathrm{KO}$ in the first 24 hours of adipocyte differentiation, although it peaked on day 2 and quickly fell to its base level while in WT preadipocytes, CREB phosphorylation gradually increased during adipocyte differentiation. This suggests that while our findings indicate that TPC2 gene expression is regulated by a mechanism involving cAMP/PKA/EPAC/CREB, there is a reciprocal regulation of CREB activity by TPC2. This raises the question of how TPC2 might be regulating CREB. One possibility, given that CREB can be phosphorylated by the $\mathrm{Ca}^{2+} /$ calmodulin-dependent kinases CaMKII and CaMKIV (Sun et al., 1994), is that $\mathrm{Ca}^{2+}$ signals induced through TPC2 influence CREB phosphorylation via one or both of these kinases. In line with this, we showed in the current study that pharmacological inhibition of CaMKII partially mimicks the effect of Tpcn $2 \mathrm{KO}$ on CREB phosphorylation. Since our findings also indicate that CREB activates Tpcn2 expression, this suggests a process of positive feedback involving TPC2 and CREB (Fig. 7).

In keeping with the results of the in vitro studies, we also demonstrate that the minor allele of a common intronic SNV at TPCN2, rs2305498, is associated with increased WHRadjBMI; a surrogate marker of body fat distribution and an independent predictor of cardiometabolic disease risk. Interestingly, the same allele is also associated with reduced TPCN2 expression in subcutaneous adipose tissue and increased estimated heel bone mineral density. Based on these findings we speculate that TPCN2 may limit adipose tissue expansion, thereby promoting upper-body fat accumulation, by altering mesenchymal stem cell fate and promoting adipogenesis at the expense of osteoblastogenesis. This hypothesis is consistent with the expression pattern of TPCN2 in fractionated human adipose tissue and the early and transient induction of Tpcn 2 gene expression during adipogenesis of C3H10T1/2 mesenchymal 
stem cells. Future, studies should focus on confirming that TPCN2 is the effector gene and adipose tissue the target tissue of the WHRadjBMI associations at the TPCN2 locus, as well, as identifying the effector cell type(s) mediating these associations. The role of TPCN2 on osteoblastogenesis should also be investigated.

\section{Materials and Methods}

Animals: Tpcn2 KO (homozygote Tpcn $2^{\mathrm{YHD} 437}$ ) mice, previously established by the Parrington/Galione labs (Department of Pharmacology, University of Oxford) (Calcraft et al, 2009b) were used. Strain-matched (C57BL/6) WT mice were used as controls. Mice were killed by cervical dislocation or asphyxiation. All experiments and procedures were approved by the local animal welfare and ethics committee of the University of Oxford, in accordance with UK guidelines for the use of experimental animals.

TD-NMR scanning: Before scanning, mice carcasses were thawed for 24 hours at $4{ }^{\circ} \mathrm{C}$ followed by 4 hours at room temperature. These samples were scanned in the same manner as live animals (Halldorsdottir et al, 2009). Previous investigations have set a precedent for MR analysis of frozen-thawed mice (Tinsley et al, 2004). Mice were weighed then scanned using a Bruker Minispec LF50 Whole Body Composition Analyser. Fat mass and lean mass data were acquired using Bruker Minispec Plus software.

Culture, treatment and adipogenic differentiation of C3H10T1/2 cells: $\mathrm{C} 3 \mathrm{H} 10 \mathrm{~T} 1 / 2$ cells (clone 9, passage 18, cells obtained from American Type Culture Collection (ATCC)) were incubated at $37^{\circ} \mathrm{C}$ with $5 \% \mathrm{CO}_{2}$ in Dulbecco's modified Eagle's medium (DMEM) (Gibco, 61965) supplemented with 10\% fetal bovine serum (FBS) and $1 \%$ penicillin/streptomycin, refreshed every second day, and passaged before exceeding $80 \%$ confluence. Differentiation was induced at 1-2 days post-confluence, designated 'day 0 '. This involved the growth medium being aspirated and replaced with complete DM (growth medium with addition of $1 \mu \mathrm{M}$ insulin, $0.5 \mathrm{mM} 3$-isobutyl1-methylxanthine (IBMX) and $1 \mu \mathrm{m}$ dexamethasone). After 2 days, this was replaced with DM/ins, and after another 2 days, this was replaced with base DM.

Differentiation was allowed to proceed for a total of 8-10 days, with the base DM refreshed every 2 days. For the untreated control condition, cells were maintained in GM, and refreshed every 2 days.

Isolation, treatment culture and differentiation of primary preadipocyte cells: Abdominal adipose tissue from mice was obtained at the age of 10-18 weeks. A Preadipocyte Isolation Kit (ab196988, Abcam) was used for preadipocyte isolation and purification according to the manufacturer's instructions. The preadipocytes were cultured in DMEM/F12, supplemented with $10 \%$ fetal bovine serum (FBS) and 1\% penicillin/streptomycin, for 48 hours until adherence. The differentiation was induced following the same standard differentiation protocol as used for $\mathrm{C} 3 \mathrm{H} 10 \mathrm{~T} 1 / 2$ cells, but replacing DMEM with DMEM/F12.

Oil red O staining: A solution of $0.2 \%$ Oil Red $\mathrm{O}(\mathrm{w} / \mathrm{v})$ in isopropanol was filtered. Cells were fixed in 4\% paraformaldehyde for $30 \mathrm{~min}$, incubated for $5 \mathrm{mins}$ in $60 \%$ 
isopropanol, then incubated in the Oil Red O solution for 10 mins before being imaged using a Leica DM 500B microscope.

AdipoRed staining and quantification: Cells were processed with AdipoRed (Lonza) reagent according to the manufacturer's instructions before being observed under a fluorescence microscope. Fluorescence was measured using a Typhoon 9400 Variable Mode Imager, with excitation of 488nm and use of a BP 580/30 emission filter. The photomultiplier tube (PMT) was adjusted to maximize the signal while avoiding saturation, and resolution was $100 \mu \mathrm{m}$. Fluorescence was quantified using Fiji/Image $\mathrm{J}$ software. Data were normalized to an average background of empty wells or, in the case of lentivirally-infected cells, to average reading for cells infected with the relevant lentivirus but not treated with AdipoRed, to control for overlap between mCherry and AdipoRed fluorescence emission.

Quantitative PCR analysis: Total RNA from C3H10T1/2 cells was isolated and purified using a QIAGEN RNeasy mini kit according to the manufacturer's instructions. The RNA concentration was checked using a NanoDrop spectrophotometer and integrity was checked by electrophoresis on a $1.5 \%$ agarose gel. cDNA was synthesized from RNA samples using a high capacity cDNA reverse transcription kit (Applied Biosystems) according to the manufacturer's instructions. The cDNA synthesized was stored at $-80^{\circ} \mathrm{C}$ until analyzed. A Roche LightCycler 480 System was used for qPCR. All primers were designed using the Roche Universal Probe Library Assay Design Centre, which also identified suitable fluorescent probes for each primer pair. A reaction mixture of cDNA $(0.00075 \mathrm{ng} / \mu \mathrm{l}-3 \mathrm{ng} / \mu \mathrm{l})$, genespecific forward and reverse primers $(0.1 \mu \mathrm{M}$ each $)$ and Universal ProbeLibrary Probe $(0.1 \mu \mathrm{M})$ in LightCycler 480 Probes Master was prepared. All samples were run in duplicate within each experiment and results averaged. Cycling conditions were $50^{\circ} \mathrm{C}$ for 2 mins; $95^{\circ} \mathrm{C}$ for 10 mins; a $30-50 \mathrm{x}$ cycle: $95^{\circ} \mathrm{C}$ for $15 \mathrm{secs} ; 60^{\circ} \mathrm{C}$ for $1 \mathrm{~min}$; then an end cycle: $40^{\circ} \mathrm{C}$ for 30 secs. LightCycler $480 \mathrm{SW} 1.5$ software acquired data and produced graphs quantifying the fluorescence of each sample plotted against cycle number and calculated the $\mathrm{Cp}$ for each sample. The value for each gene of interest was normalized to the averaged housekeeping gene value and were expressed as values relative to a control ( 0 hour timepoint for differentiation time-course experiments, GM or vehicle control for pharmacological interventions).

Lentiviral expression of TPC2: Lenti-X HEK293T cells (Clontech) were grown at $37^{\circ} \mathrm{C}, 5 \% \mathrm{CO}_{2}$ in DMEM supplemented with $10 \%$ tetracycline-free foetal calf serum (BioSera), $1 \mathrm{mM}$ sodium pyruvate, $100 \mathrm{U} / \mathrm{ml}$ penicillin-G-sodium, $100 \mu \mathrm{g} / \mathrm{ml}$ streptomycin sulphate and $2 \mathrm{mM}$ L-glutamine. When $50-80 \%$ confluent, cells were co-transfected with viral vector and Lenti-X HT packaging mix (Clontech, 15 $\mu 1$ per $3 \mu \mathrm{g}$ vector) using a jetPEI system (Polyplus transfection) according to the manufacturer's instructions. Medium was harvested 48 hours after transfection, filtered through a sterile $0.45 \mu \mathrm{m}$ polyethersulfonate filter (Whatman puradisc $25 \mathrm{AS}$ ), and checked for the presence of infectious virons by applying a sample to Lenti-X GoStix (Clontech). When cells reached 50\% confluence, mCherry, mCherry-tagged TPC lentiviral medium or control medium was added ( $1 \mathrm{ml} / \mathrm{T} 75$ flask) along with polybrene to $4 \mu \mathrm{g} / \mathrm{ml}$ final concentration. 
Fluorescence and immunofluorescence imaging: C3H10T1/2 cells (seeded on coverslips) were washed in PBS and fixed for 15 mins in $4 \%$ paraformaldehyde, then mounted with ProLong Gold antifade mounting medium (Invitrogen) (for mCherry fluorescence observation) or processed for immunofluorescence: cells were permeabilised in PBS/0.5\% Triton X, then blocked in 2\% goat serum followed by incubation in 5F8 monoclonal against mCherry, supplied by A. Rottach, University of Munich, diluted 1:500, for 1 hour at room temperature, and incubation in goat anti-rat Alexa Fluor 488 (Invitrogen), $5 \mu \mathrm{g} / \mathrm{ml}$. Coverslips were mounted and slides viewed using a Zeiss Meta 510 confocal LSM.

Immunoblot analysis: Cells were lysed by RIPA (Sigma) and dissolved in $5 \times$ Laemmli sample buffer (ThermoFisher) supplemented with phosphatase inhibitor cocktails (Sigma). The proteins were boiled at $96^{\circ} \mathrm{C}$ for 5 mins, separated by SDSpolyacrylamide gel electrophoresis (SDS-PAGE), and transferred to polyvinylidene difluoride membranes. To block nonspecific binding sites, membranes were incubated in Tris-buffered saline containing $0.1 \%(\mathrm{v} / \mathrm{v})$ Tween 20 and 5\% (w/v) skim milk powder before primary antibodies of the specificity of interest were added. Films were observed by LI-COR Odyssey 9120 Digital Imaging System.

Calpain activity analysis: Calpain activity of differentiating primary preadipocytes was measured using a Calpain Activity Assay Kit (ab65308) according to the manufacturer's instructions. Fluorescence was read by a Tecan M200 Pro microplate reader.

CREB phosphorylation analysis: Cells were subject to same lysis procedure as for Western Blot analysis. CREB levels (total/phosphorylated) were measured using a CREB InstantOne ELISA kit (Invitrogen) according to the manufacturer's instructions.

Analysis of TPCN2 genetic variants in humans: Abdominal and gluteal subcutaneous adipose tissue biopsies were obtained from 11 healthy females recruited from the Oxford BioBank (OBB), aged 30-50 years with BMI ranging from $22-27 \mathrm{~kg} / \mathrm{m}^{2}$. The study was approved by Oxfordshire Clinical Research Ethics Committee $(08 / \mathrm{H} 0606 / 107+5)$ and all subjects gave written informed consent. Stromal vascular fraction (SVF) cells and mature adipocytes were separated from whole adipose tissue biopsies following collagenase (Roche) digestion ( $1 \mathrm{mg} / \mathrm{ml}$ in Hanks' buffered salt solution) and in the case of SVF cells additional treatment with red cell lysis buffer. Total RNA was extracted as described by Collins et al (Collins et al, 2010). RNA integrity was assessed using a 2100 Bioanalyzer (Agilent Technologies) and samples with a RNA integrity (RIN) number of 8 and above were selected for RNASeq analysis. These samples were sent to the the Wellcome Trust Centre for Human Genetics where a quality control report was generated and a library of read data compiled. Differentially expressed genes were identified by comparing the RNAseq profiles between SVF, mature adipocytes and to the whole adipose tissue samples.

Statistical analysis: All data are presented as +/- standard error of the mean (SEM). For most data comparisons, unpaired 2-tail student's t-test was used $\left({ }^{*} p<0.05\right.$; $* * \mathrm{p}<0.01 ; * * * \mathrm{p}<0.001)$. For multiple comparison of 3 or more data sets, one-way 
analysis of variance (ANOVA) was used with post-hoc Tukey test (\#\#p<0.01; $\# \# \# p<0.001)$. For statistical analysis of variation in gene expression and calpain activity during adipogenic differentiation time-course, one-way ANOVA was used with a post-hoc Dunnett test comparing all timepoints to expression at time zero $(\S p<0.05 ; \S \S p<0.01)$. Statistical analyses were conducted using GraphPad Prism8.

\section{Author Contributions}

JP, YZ, LHC and RT designed and performed experiments, analyzed all data, and wrote the manuscript. MR, DG, MT and CC performed experiments and helped to write the manuscript.

\section{Declaration of Interests}

All authors declare no competing interests. 


\section{References}

Ahmed A, Boulton S, Shao H, Akimoto M, Natarajan A, Cheng X \& Melacini G (2019) Recent Advances in EPAC-Targeted Therapies: A Biophysical Perspective. Cells 8: 1462

Aimes RT, Hemmer W \& Taylor SS (2000) Serine-53 at the Tip of the Glycine-Rich Loop of cAMP-Dependent Protein Kinase: Role in Catalysis, P-Site Specificity, and Interaction with Inhibitors ${ }^{\dagger}$. Biochemistry 39: 8325-8332

Ali AT, Hochfeld WE, Myburgh R \& Pepper MS (2013) Adipocyte and adipogenesis. European Journal of Cell Biology 92: 229-236

Berridge MJ, Bootman MD \& Roderick HL (2003) Calcium signalling: dynamics, homeostasis and remodelling. Nat Rev Mol Cell Biol 4: 517-529

Bonnefond M-L, Lambert B, Giffard F, Abeilard E, Brotin E, Louis M-H, Gueye MS, Gauduchon P, Poulain L \& N'Diaye M (2015) Calcium signals inhibition sensitizes ovarian carcinoma cells to anti-Bcl-xL strategies through Mcl-1 down-regulation. Apoptosis 20: 535-550

Brailoiu E, Churamani D, Cai X, Schrlau MG, Brailoiu GC, Gao X, Hooper R, Boulware MJ, Dun NJ, Marchant JS, et al (2009) Essential requirement for two-pore channel 1 in NAADP-mediated calcium signaling. J Cell Biol 186: 201-209

Calcraft PJ, Ruas M, Pan Z, Cheng X, Arredouani A, Hao X, Tang J, Rietdorf K, Teboul L, Chuang K-T, et al (2009a) NAADP mobilizes calcium from acidic organelles through two-pore channels. Nature 459: 596-600

Calcraft PJ, Ruas M, Pan Z, Cheng X, Arredouani A, Hao X, Tang J, Rietdorf K, Teboul L, Chuang K-T, et al (2009b) NAADP mobilizes calcium from acidic organelles through two-pore channels. Nature 459: 596-600

Cheng X, Ji Z, Tsalkova T \& Mei F (2008) Epac and PKA: a tale of two intracellular cAMP receptors. Acta Biochimica et Biophysica Sinica 40: 651-662

Collins JM, Neville MJ, Hoppa MB \& Frayn KN (2010) De novo lipogenesis and stearoyl-CoA desaturase are coordinately regulated in the human adipocyte and protect against palmitate-induced cell injury. J Biol Chem 285: 6044-6052

Engin A (2017) Fat Cell and Fatty Acid Turnover in Obesity. In Obesity and Lipotoxicity, Engin AB \& Engin A (eds) pp 135-160. Cham: Springer International Publishing

Galione A (2011) NAADP Receptors. Cold Spring Harbor Perspectives in Biology 3: a004036-a004036

Galione A \& Chuang K-T (2020) Pyridine Nucleotide Metabolites and Calcium Release from Intracellular Stores. In Calcium Signaling, Islam MdS (ed) pp 371-394. Cham: Springer International Publishing 
Galione A \& Churchill GC (2000) Cyclic ADP Ribose as a Calcium-Mobilizing Messenger. Science Signaling 2000: pe1-pe1

Galione A \& Churchill GC (2002) Interactions between calcium release pathways: multiple messengers and multiple stores. Cell Calcium 32: 343-354

GTEx Portal (https://www.gtexportal.org/home/) [PREPRINT]

Halldorsdottir S, Carmody J, Boozer CN, Leduc CA \& Leibel RL (2009) Reproducibility and accuracy of body composition assessments in mice by dual energy x-ray absorptiometry and time domain nuclear magnetic resonance. Int J Body Compos Res 7: 147-154

Haslam RJ, Davidson MML \& Desjardins JV (1978) Inhibition of adenylate cyclase by adenosine analogues in preparations of broken and intact human platelets. Evidence for the unidirectional control of platelet function by cyclic AMP. Biochemical Journal 176: 83-95

Jin X, Zhang Y, Alharbi A, Hanbashi A, Alhoshani A \& Parrington J (2020) Targeting Two-Pore Channels: Current Progress and Future Challenges. Trends Pharmacol Sci 41: 582-594

Kelly MP (2018) Cyclic nucleotide signaling changes associated with normal aging and age-related diseases of the brain. Cell Signal 42: 281-291

Laurenza A, Sutkowski EM \& Seamon KB (1989) Forskolin: a specific stimulator of adenylyl cyclase or a diterpene with multiple sites of action? Trends Pharmacol Sci 10: 442-447

Liu L \& Clipstone NA (2007) Prostaglandin F2 $\alpha$ inhibits adipocyte differentiation via a Gaq-Calcium-Calcineurin-Dependent signaling pathway. J Cell Biochem 100: $161-173$

Ma H, Groth RD, Cohen SM, Emery JF, Li B, Hoedt E, Zhang G, Neubert TA \& Tsien RW (2014) $\gamma$ CaMKII Shuttles Ca2+/CaM to the Nucleus to Trigger CREB Phosphorylation and Gene Expression. Cell 159: 281-294

Morgan AJ, Davis LC, Wagner SKTY, Lewis AM, Parrington J, Churchill GC \& Galione A (2013) Bidirectional Ca2+ signaling occurs between the endoplasmic reticulum and acidic organelles. The Journal of Cell Biology 200: 789-805

Neal JW \& Clipstone NA (2002) Calcineurin Mediates the Calcium-dependent Inhibition of Adipocyte Differentiation in 3T3-L1 Cells. J Biol Chem 277: 49776-49781

Ntambi JM \& Takova T (1996) Role of Ca2+ in the early stages of murine adipocyte differentiation as evidenced by calcium mobilizing agents. Differentiation 60: $151-158$

Patel YM \& Lane MD (1999) Role of calpain in adipocyte differentiation. Proceedings of the National Academy of Sciences 96: 1279-1284 
Pulit SL, Stoneman C, Morris AP, Wood AR, Glastonbury CA, Tyrrell J, Yengo L, Ferreira T, Marouli E, Ji Y, et al (2019) Meta-analysis of genome-wide association studies for body fat distribution in 694649 individuals of European ancestry. Hum Mol Genet 28: 166-174

Ruas M, Davis LC, Chen C, Morgan AJ, Chuang K, Walseth TF, Grimm C, Garnham C, Powell T, Platt N, et al (2015) Expression of $\mathrm{Ca}^{2+}$-permeable two-pore channels rescues NAADP signalling in TPC -deficient cells. EMBO J 34: $1743-1758$

Ruas M, Rietdorf K, Arredouani A, Davis LC, Lloyd-Evans E, Koegel H, Funnell TM, Morgan AJ, Ward JA, Watanabe K, et al (2010) Purified TPC Isoforms Form NAADP Receptors with Distinct Roles for Ca2+ Signaling and Endolysosomal Trafficking. Current Biology 20: 703-709

Sarjeant K \& Stephens JM (2012) Adipogenesis. Cold Spring Harbor Perspectives in Biology 4: a008417-a008417

Taylor SM \& Jones PA (1979) Multiple new phenotypes induced in and 3T3 cells treated with 5-azacytidine. Cell 17: 771-779

Thompson WJ (1991) Cyclic nucleotide phosphodiesterases: Pharmacology, biochemistry and function. Pharmacology \& Therapeutics 51: 13-33

Tinsley FC, Taicher GZ \& Heiman ML (2004) Evaluation of a Quantitative Magnetic Resonance Method for Mouse Whole Body Composition Analysis. Obesity Research 12: 150-160

Xie F, Fan Q, Li BX \& Xiao X (2019) Discovery of a Synergistic Inhibitor of cAMPResponse Element Binding Protein (CREB)-Mediated Gene Transcription with 666 - 15. J Med Chem 62: 11423-11429

Yajima Y, Sato M, Sorimachi H, Inomata M, Maki M \& Kawashima S (2006) Calpain System Regulates the Differentiation of Adult Primitive Mesenchymal ST-13 Adipocytes. Endocrinology 147: 4811-4819

Zhang F, Ye J, Meng Y, Ai W, Su H, Zheng J, Liu F, Zhu X, Wang L, Gao P, et al (2018) Calcium Supplementation Enhanced Adipogenesis and Improved Glucose Homeostasis Through Activation of Camkii and PI3K/Akt Signaling Pathway in Porcine Bone Marrow Mesenchymal Stem Cells (pBMSCs) and Mice Fed High Fat Diet (HFD). Cell Physiol Biochem 51: 154-172 


\section{Figure Legends}

\section{Figure 1- TPC2 mRNA expression is up-regulated transiently during adipocyte differentiation in vitro}

A AdipoRed and Oil Red O staining of differentiating C3H10T1/2 cells, immediately before start of differentiation induction with adipogenic medium, and 4 and 8 days after the start of treatment. Accumulation of the stain indicates lipid droplet formation. Scale bar: $100 \mu \mathrm{m}$

B mRNA levels of adipogenic marker genes in differentiating C3H10T1/2 cells. ( a) Ppary2; (b) Srebp1; (c) aP2; (d) c/Ebp $\delta . \mathrm{n}=2$ (c/Ebp $\delta) ; \mathrm{n}=3$ (Ppary2, aP2, Srebp1).

C Tpcn 2 mRNA levels in differentiating C3H10T1/2 cells. $\mathrm{n}=3$.

D C3H10T1/2 differentiation medium components. (FCS: fetal calf serum)

Data information: In (B), data are presented as mean \pm SEM, values are normalised to the geometric mean of $18 \mathrm{~S}$ and ubiquitin $\mathrm{c}$ mRNA expression and presented as foldchange relative to $0 \mathrm{~h}$ value. $\S \mathrm{p}<0.05 ; \S \S \mathrm{p}<0.01$ (ANOVA, post-hoc Dunnett test vs $0 \mathrm{~h}$ ). In (C), data are presented as mean \pm SEM, $\S \S p<0.01$ (ANOVA, post-hoc Dunnett test vs $0 \mathrm{~h}$ )

\section{Figure 2 - Tpcn 2 mRNA expression during adipocyte differentiation}

A Determination of the component(s) of the adipogenic differentiation medium that stimulates Tpcn 2 mRNA expression. $\mathrm{n}=3$. Grey bar: sample at 4 hours, $\mathrm{n}=1$.

B Effect of IBMX on Tpcn2 mRNA expression in 2-day post-confluent C3H10T1/2 cells. $n=3$.

C Expression of Tpcn 2 mRNA in C3H10T1/2 cells in response to drugs that alter cAMP levels. Cells were treated for 4 hours at 2 days post-confluence with GM (growth media) containing 50 $\mu \mathrm{M}$ forskolin (in DMSO), $100 \mu \mathrm{M}$ rolipram (in DMSO), $500 \mu \mathrm{M}$ IBMX (in $100 \%$ ethanol) or vehicle. $\mathrm{n}=3$.

D Effect of the adenylyl cyclase inhibitor SQ22536 on IBMX-induced induction of Tpcn 2 mRNA expression. 2-day post-confluent cells were preincubated for 30 minutes with GM containing $300 \mu \mathrm{M}$ SQ22536 or vehicle (water) before $500 \mu \mathrm{M}$ IBMX was added. $n=3$.

E, F, G Comparison of Tpcn2 mRNA in non-treated (control) and (E) PKI (PKA inhibitor) / (F) ESI-09 (Epac inhibitor) / (G) 666-15 (CREB inhibitor) treated C3H10T1/2 cells. At 2 days post confluence, $10 \mu \mathrm{M}$ PKI / $25 \mu \mathrm{M}$ ESI-09/ $1 \mu \mathrm{M}$ 66615 were added to the medium during the entire time course of differentiation. $n=5-8$,

Data information: Data are presented as mean \pm SEM. In (A-D), values are normalised to the geometric mean of $18 S$ and ubiquitin $c$ mRNA expression and presented as fold-change relative to GM condition. In (A), \#\#\#p<0.001, (ANOVA,

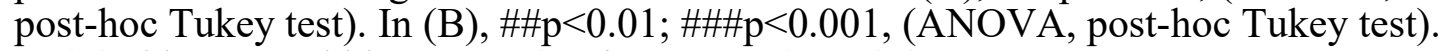

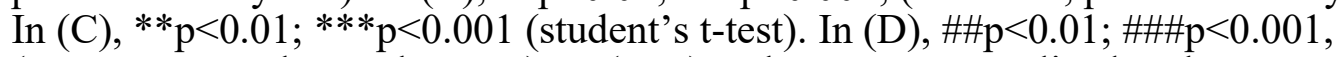
(ANOVA, post-hoc Tukey test). In (E-G), values were normalized to the geometric mean of $\beta$-Actin mRNA expression and are presented as expression relative to $0 \mathrm{hr}$. $* * \mathrm{p}<0.01$ (student's t-test).

Figure 3- TPC2 plays a functional role during adipocyte differentiation in vitro 
A Anti-mCherry western blot confirming expression of lentivirally-delivered mCherry-tagged TPC2 in C3H10T1/2 cells (a); anti-mCherry western blot confirming expression of lentivirally-introduced mCherry (b).

B Confocal images of mCherry fluorescence (red) and anti-mCherry immunofluorescence (green) in C3H10T1/2 cells expressing lentivirally-introduced mCherry and mCherry tagged TPCs. Scale bar: $30 \mu \mathrm{m}$.

C AdipoRed quantification of adipogenesis in $\mathrm{C} 3 \mathrm{H} 10 \mathrm{~T} 1 / 2$ cells lentivirally infected with mCherry or mCherry-tagged TPC2. Cells were treated according to standard differentiation medium protocol (complete DM); with standard protocol omitting IBMX (DM-IBMX); or maintained in growth medium (GM), and were assayed after 8 days. $n=8-12$.

D AdipoRed quantification of adipogenesis in primary preadipocyte cells from WT and Tpcn $2 \mathrm{KO}$ mice treated with standard differentiation protocol and control group maintained in growth medium, assayed after 8 days. $\mathrm{n}=6$.

Data information: In (C, D), data are presented as mean \pm SEM. ${ }^{* *} \mathrm{p}<0.01$, $* * * \mathrm{p}<0.001$ (student's t-test).

\section{Figure 4 - Calpain activity during adipocyte differentiation}

A, B Variation in calpain activity in the process of primary preadipocyte differentiation. (A) WT preadipocytes. $\mathrm{n}=8$. (B) Tpcn $2 \mathrm{KO}$ preadipocytes. $\mathrm{n}=3-8$.

Data information: In (A, B), data are presented as mean \pm SEM. \#\#\#p $<0.001$ (ANOVA, post-hoc Tukey test, compared between consecutive time points).

\section{Figure 5 - CREB phosphorylation during adipocyte differentiation}

A, B, C Levels of CREB and phosphorylated CREB during preadipocyte differentiation. (A) WT preadipocytes. $\mathrm{n}=3$. (B) Tpcn $2 \mathrm{KO}$ preadipocytes. $\mathrm{n}=3$. (C) WT preadipocytes treated with KN 93.

D Ratio of phosphorylated CREB to total CREB during adipocyte differentiation.

Data information: In (A-C), data are presented as mean \pm SEM. $\S \S \S p<0.001$ (one-way ANOVA was used with post-hoc Dunnett test comparing all timepoints to expression at $0 \mathrm{~h})$.

\section{Figure 6 - Genetic variation at the TPCN2 locus is associated with altered fat distribution}

A TPCN2 is most highly expressed in adipose compared with other metabolic tissues. RNA-Seq data from GTex.

B TPCN2 expression in whole and fractionated adipose tissue. RNA-Seq data from 11 females. AT, adipose tissue, mADS, mature adipocytes, SVF, stomovascular fraction, TPM, transcripts per million. ${ }^{* * *} \mathrm{p} \leq 0.0001$, two-tailed paired Student's t-test. 
C Locus zoom showing a common intronic SNV at TPCN2, rs2305498, is associated with increased waist-hip ratio adjusted for BMI.

\section{Figure 7 - Key components modulators in cAMP signaling and adipogenesis.}

Schematic showing key components with their pharmacological modulators in cAMP signaling pathway and how they regulate adipogenesis. Figure created with BioRender.com. (AC=adenylyl cyclase; $\mathrm{PDE}=$ phosphodiesterase; $\mathrm{CREB}=\mathrm{cAMP}-$ response element binding protein). 


\section{Expanded view}

\section{Tpen2 KO mice of both sexes have abnormal adiposity compared to wild type controls}
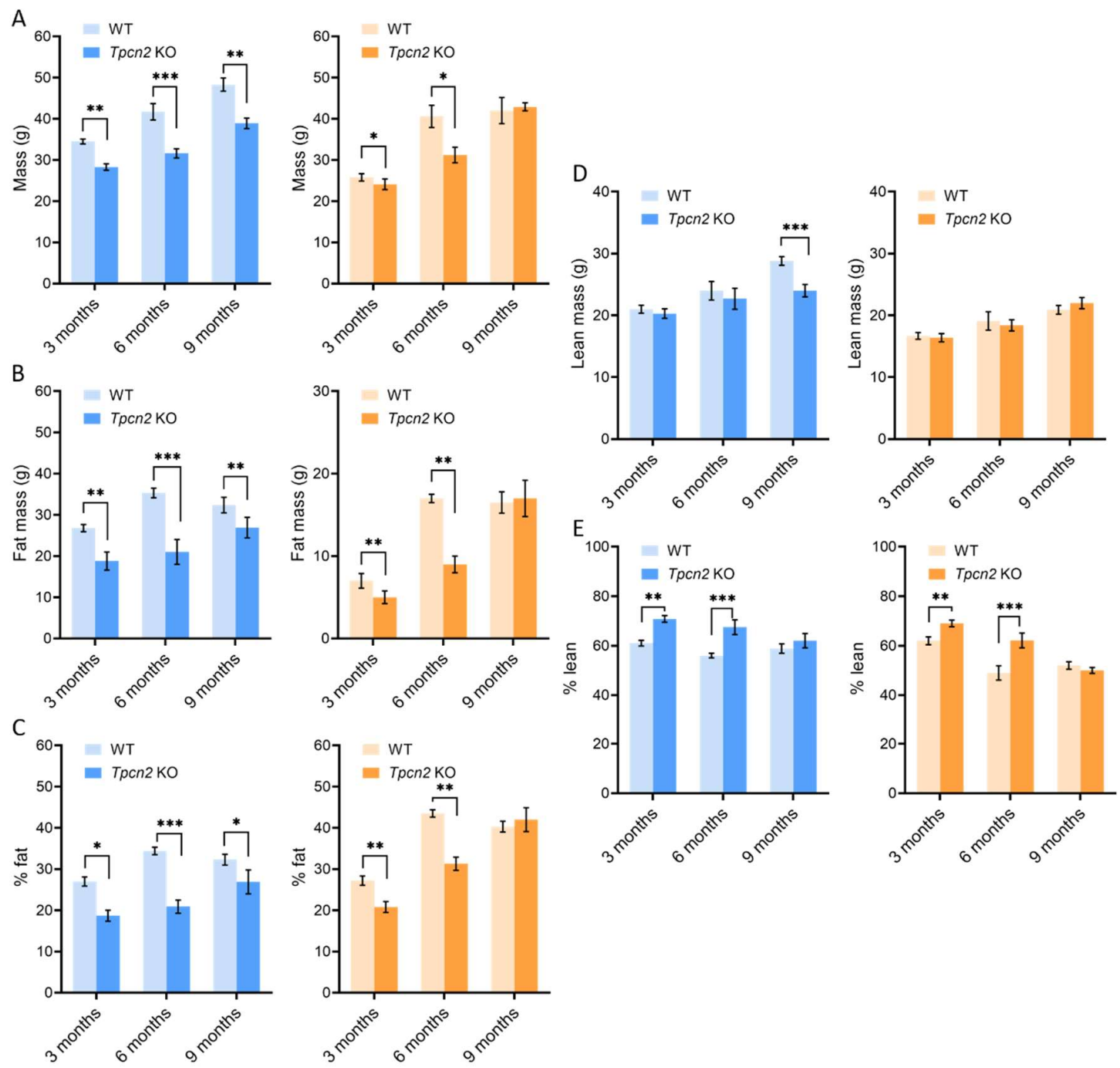

\section{Figure EV1}

Male (blue) and female (orange) Tpcn $2 \mathrm{KO}$ and WT body composition quantified by TD-NMR:

A) total body mass; B) fat mass; C) percentage fat; D) lean mass; E) percentage lean. $n=7-8$. ${ }^{*} \mathrm{p}<0.05 ; * * \mathrm{p}<0.01 ; * * * \mathrm{p}<0.001$ (student's t-test).

\section{Intraperitoneal glucose tolerance test (IPGTT) of TPC KO mice}

In response to a glucose challenge, Tpcn2 $\mathrm{KO}$ mice showed a slight, non-significant tendency towards blood glucose peaking higher than in WTs, with no significant difference in total glycaemia. 

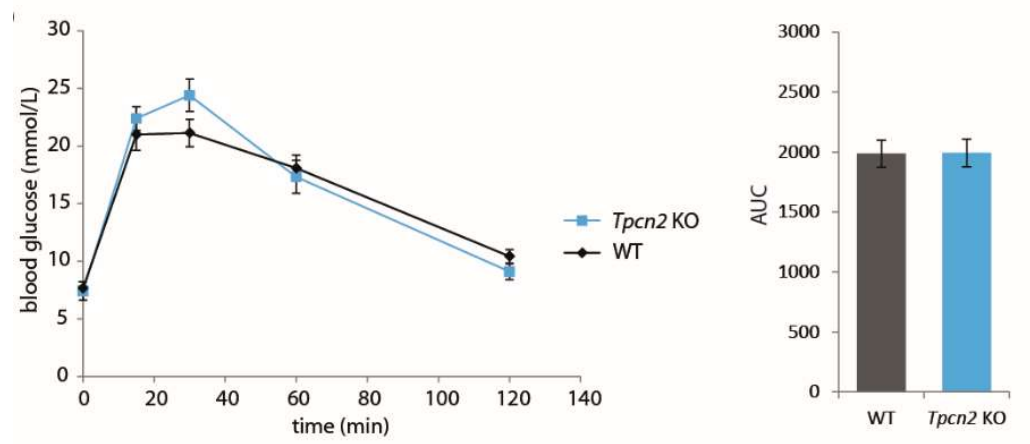

Figure EV2: Intraperitoneal glucose tolerance test (IPGTT). Response to $2 \mathrm{~g} / \mathrm{kg}$ IP glucose following overnight fast in male Tpcn $2 \mathrm{KO}$ and strain-matched WT mice aged 9-11 weeks. Tpcn2 $\mathrm{KO}(\mathrm{n}=11), \mathrm{WT}(\mathrm{n}=8)$; student's t-test. AUC $=$ area under the curve.

\section{Anti-mCherry immunofluorescence}

Figure S2 shows anti-mCherry immunofluorescence in MEFs infected with lentiviruses bearing cDNA encoding mCherry and mCherry-tagged TPC2. Anti-mCherry immunofluorescence was clearly visible in infected cells, corresponding to obvious mCherry fluorescence in mCherrytagged TPC 2 infected cells. The expression of mCherry-tagged TPC 2 would be predicted to also be punctate based on the previously observed lysosomal localization of TPC2, but this was less obvious - possibly due to excessive overexpression, or simply to overexposure of the confocal images. mCherry fluorescence in cells infected with mCherry only was less bright and more difficult to distinguish from background but was confirmed as genuine mCherry fluorescence by colocalization with anti-mCherry immunofluorescence.

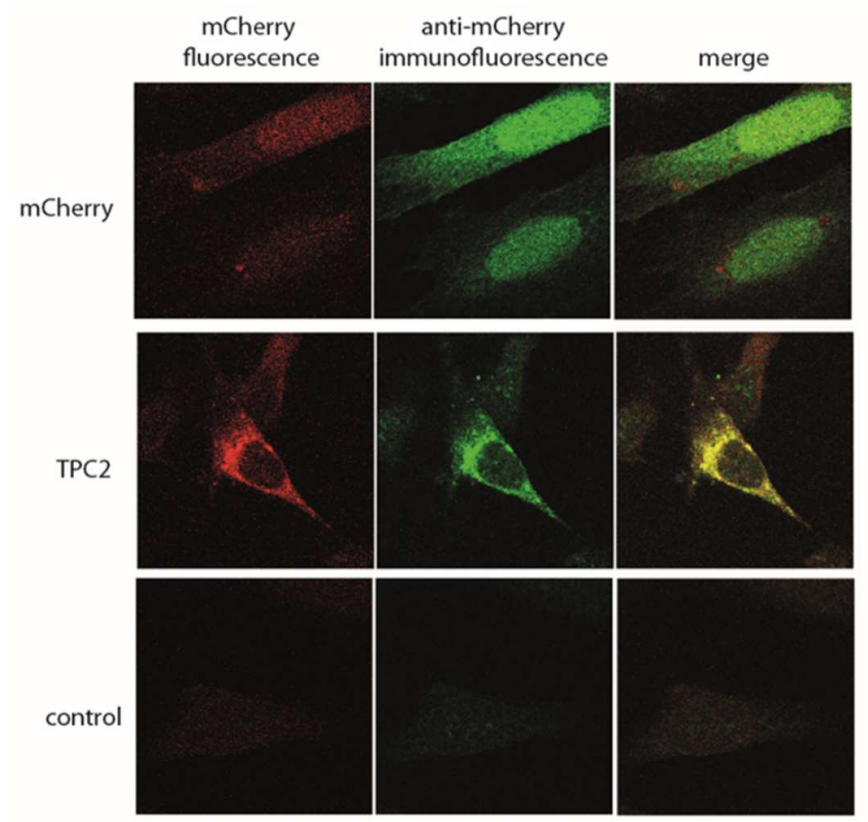

Figure EV3: Expression of lentivirally-transduced mCherry and mCherry-tagged TPC2 protein in paraformaldehyde-fixed MEFs. mCherry fluorescence (red); anti-mCherry immunofluorescence (green). 
Table EV1: List of oligonucleotides used for qPCR.

\begin{tabular}{|l|l|l|}
\hline Mus Gene & Forward primer sequence & Reverse primer sequence \\
\hline Tpcn 2 & CCCTGGCTGTATACCGATTG & GTCCCAGAGCGACAGTGG \\
\hline$a P 2$ & AAGAGAAAACGAGATGGTGACAA & CTTGTGGAAGTCACGCCTTT \\
\hline c/Ebp $\delta$ & TTCAACAGCAACCACAAAGC & CTAGCGACAGACCCCACAC \\
\hline Ppary 2 & GAAAGACAACGGACAAATCACC & GGGGGTGATATGTTTGAACTTG \\
\hline Srebp 1 & ACAAGATTGTGGAGCTCAAAGAC & GCGCAAGACAGCAGATTTATT \\
\hline $18 \mathrm{~S}$ & CTCAACACGGGAAACCTCAC & CGCTCCACCAACTAAGAACG \\
\hline$\beta$-Actin & CTCAACACGGGAAACCTCAC & CGCTCCACCAACTAAGAACG \\
\hline
\end{tabular}

